\title{
Pérdidas Proteicas Peritoneales en Niños Portadores de Síndrome Nefrótico en Diálisis Peritoneal
}

\author{
LILY QUIROZ Z.', MARTA AZÓCAR P. ${ }^{1}$, HÉCTOR DINAMARCA S. ${ }^{1}$, \\ MARCOS EMILFORK S. ${ }^{2}$, FRANCISCO CANO SCH. ${ }^{1}$ \\ 1. Facultad de Medicina, Universidad de Chile, Hospital Luis Calvo Mackenna, Santiago, Chile. \\ 2. Servicio de Pediatría, Clínica Santa María, Santiago, Chile.
}

\begin{abstract}
Peritoneal Protein Loss in Children Suffering from Nephrotic Syndrome Undergoing Peritoneal Dialysis

Primary Nephrotic Syndrome (NS) responds favorably to steroids in $80-90 \%$ of cases. Most corticoresistant (CR) patients evolve into Chronic Renal Failure (CRF), Of unknown origin, a permeability factor in these patient's serum has been reported, with some known effects in membranes including the peritoneum. Objective: To evaluate peritoneal protein loss in CR children on Chronic Peritoneal Dialysis (CPD). Patients and Methods: Four year retrospective analysis. Group 1 included 9 CR children, Group 2 was a control group of 10 children with CRF of other causes on CPD. Children in both groups were comparable in age, gender, weight, body surface, duration of CPD, concentration of solution, modality and dose of dialysis. Both groups were evaluated at 1, 6 and 12 months after admission. Results: No differences were observes in biochemical parameters: creatinine, urea nitrogen, calcium, phosphorus. PTH (Parathyroid hormone) was significantly higher in the control group ( $164 \pm 144$ vs $564 \pm 454 \mathrm{pg} / \mathrm{dl} \mathrm{p}<0,05)$, albumin was lower in NS patients at the beginning $(2.27 \pm 0.63 \mathrm{gr} / \mathrm{dl} v s 3.62 \pm 1.45 \mathrm{gr} / \mathrm{dl} \mathrm{p}<0,05)$ and end $(2.8 \pm 0.5 \mathrm{gr} /$ $\mathrm{dl} v s 3.9 \pm 0.86 \mathrm{gr} / \mathrm{dl}, \mathrm{p}<0,05)$ of the evaluation. Peritoneal protein loss was significantly larger in the index group at the beginning $\left(3,41 \pm 2,01 v s 1,76 \pm 1,45 \mathrm{gr} / \mathrm{m}^{2} / \mathrm{dí}\right)$ ), and end $\left(4,27 \pm 3,47 v s 1,66 \pm 1,31 \mathrm{gr} / \mathrm{m}^{2} / \mathrm{dí}\right.$, (p $<0.05)$ of the evaluation. The same happened with urinary loss: while there was no difference in protein intake, peritoneal $\mathrm{KtV}$ or total $\mathrm{KtV}$ between groups, residual $\mathrm{KtV}$ was significantly lower among NS patients at the end of the study, suggesting an earlier drop in residual renal function. No differences were observed in rates of peritonitis between groups in the study period. Conclusion: Peritoneal protein loss in CPD children with NS are significantly larger than other patients in CPD, suggesting a possible systemic permeability factor in these patients.
\end{abstract}

(Key words: Nephrotic syndrome, peritoneal protein loss, children, permeability factor, dialysis).

Rev Chil Pediatr 2009; 80 (5): 427-434

Trabajo recibido el 21 de abril de 2009, devuelto para corregir el 05 de agosto de 2009, segunda versión el 06 de agosto de 2009, aceptado para publicación el 10 de agosto de 2009.

Trabajo distinguido con el Premio de la Sociedad Chilena de Pediatría al mejor trabajo de Investigación calidad Becado de Pediatría, año 2008.

Correspondencia a:

Dra. Lily Quiroz Z.

E-mail: lilyqzz@gmail.com 


\section{RESUMEN}

El Síndrome Nefrótico primario (SN) responde favorablemente a corticoides en un $80-90 \%$ de los casos. Los pacientes cortico resistentes (SNCR) evolucionan, en su gran mayoría, a insuficiencia renal crónica (IRC). De etiología desconocida, se ha reportado la presencia de un factor de permeabilidad (FP) en el suero de estos pacientes, con algunos efectos conocidos a nivel de otras membranas biológicas, incluyendo el peritoneo. Objetivo: Evaluar las pérdidas proteicas vía peritoneo en niños con SNCR en diálisis peritoneal crónica (DP). Pacientes y Método: Análisis retrospectivo de 4 años (2003-2007), Se incluyeron 9 pacientes portadores de SNCR (grupo 1), y un grupo control de 10 niños en DP portadores de IRC por otra etiología (grupo 2). Se evaluó a los 2 grupos al mes 1 y 6 ó 12 de su ingreso. Los grupos fueron comparables respecto a edad, sexo, peso, superficie corporal, tiempo en DP, concentración de dextrosa utilizada, modalidad dialítica y dosis de diálisis. Resultados: No se observó diferencias de los parámetros bioquímicos (creatinina, nitrógeno ureico, calcio, fósforo). La hormona paratiroidea (PTH) fue significativamente mayor en el grupo control $(164 \pm 144$ vs $564 \pm 454 \mathrm{pg} / \mathrm{dl} \mathrm{p}<0,05)$, y la albúmina fue menor en los pacientes con SN al inicio $(2,27 \pm 0,63 \mathrm{gr} / \mathrm{dl} v s 3,62 \pm 1,45 \mathrm{gr} / \mathrm{dl} \mathrm{p}<0,05)$ y al final de la evaluación $(2,8 \pm 0,5 \mathrm{gr} / \mathrm{dl} v s 3,9 \pm 0,86 \mathrm{gr} / \mathrm{dl}$, $\mathrm{p}<0,05$ ). Las pérdidas proteicas peritoneales fueron significativamente mayores en el grupo $1 v \mathrm{~s}$ el grupo 2 al ingreso: $3,41 \pm 2,01$ vs $1,76 \pm 1,45 \mathrm{gr} / \mathrm{m}^{2} /$ día, y al final de la evaluación: $4,27 \pm 3,47 v s 1,66 \pm 1,31 \mathrm{gr} / \mathrm{m}^{2} / \mathrm{dí}^{2}$, $(\mathrm{p}<0,05)$ respectivamente. Lo mismo ocurrió con las pérdidas urinarias. No hubo diferencias en la ingesta proteica, $\mathrm{KtV}$ peritoneal ni $\mathrm{KtV}$ total entre los grupos, mientras que el $\mathrm{KtV}$ residual fue significativamente menor en los pacientes nefróticos al término del estudio, sugiriendo una caída más precoz de la función renal residual. Tampoco se observó diferencias respecto a las tasas de peritonitis en el período estudiado. Conclusión: Las pérdidas de proteínas por peritoneo en pacientes en DP portadores de SN, son significativamente mayores que en el resto de los pacientes en DP, y son consistentes con un posible efecto sistémico de un FP en estos pacientes.

(Palabras clave: Síndrome nefrótico, factor de permeabilidad, diálisis, peritoneo).

Rev Chil Pediatr 2009; 80 (5): 427-434

\section{Introducción}

El Síndrome Nefrótico (SN) es una enfermedad caracterizada por edema, hipoalbuminemia, proteinuria masiva e hiperlipidemia. Afecta niños principalmente de 2 a 8 años con una incidencia de 2 a 7 casos por 100000 niños y una prevalencia de 16 casos por 100000 habitantes, siendo mayor en poblaciones asiáticas y afro-americanas ${ }^{1-3}$. La glomerulopatía asociada más frecuente corresponde a los cambios mínimos glomerulares (76\%), de buen pronóstico, en contraposición con la glomeruloesclerosis focal y segmentaria (GEFS) (8\%), en que un importante porcentaje de ellos no responde al tratamiento inmunosupresor y llegan a insuficiencia renal crónica (IRC) terminal ${ }^{4}$. La literatura ha reportado un incremento en la incidencia de GEFS tanto en pacientes adultos como pediátricos. Hace 30 años la incidencia de GEFS en biopsias de niños era 7\%, siendo en reportes más recientes hasta $12-35 \%$ de los $\operatorname{casos}^{4-8}$. En pacientes pediátricos en diálisis crónica, el 15-
$20 \%$ presenta síndrome nefrótico como etiología de su IRC ${ }^{9}$, y alrededor de un $14 \%$ de éstos corresponden a GEFS ${ }^{10}$.

Una observación frecuente en los pacientes portadores de $\mathrm{SN}$ en diálisis peritoneal, es el persistente compromiso nutricional, aún por sobre la malnutrición usualmente observada en IRC. La hipoalbuminemia y la hipercolesterolemia son elementos que se observan persistentemente y obligan a un estricto manejo hidroelectrolítico y de ultrafiltración, lo cual hace de estos pacientes un grupo especialmente difícil de manejar y de reservado pronóstico ${ }^{11-14}$. Los inhibidores de la enzima convertidora (IECA), los antagonistas de angiotensina II, la ultrafiltración agresiva e incluso la nefrectomía uni o bilateral, han sido usadas en el manejo de estos pacientes, con una respuesta poco predecible y a veces insuficiente ${ }^{15-18}$. De especial interés resulta constatar la persistencia de hipoalbuminemia en pacientes con un $\mathrm{Kt} / \mathrm{V}$ residual $<0,1$. Estas observaciones han sugerido la hipótesis sobre la existencia de pérdidas 
proteicas adicionales a las que se presentan por vía urinaria, insinuando la existencia de un factor sistémico de permeabilidad capilar que afectaría la homeostasis proteica en estos pacientes ${ }^{19}$.

En 1974 Shaloub describió la presencia de una linfokina presente en el suero de pacientes con SN idiopático que actuaba sobre el capilar glomerular favoreciendo la pérdida de proteínas por orina, denominando a esta molécula "Factor de Permeabilidad"18. Posteriormente, se postuló que este factor de permeabilidad podría afectar otras membranas distintas a la membrana basal glomerular, afectando su permeabilidad, es así como el año 2006 Kopanati publicó su experiencia realizada en 60 pacientes pediátricos en DP comparando las pérdidas proteicas peritoneales de un grupo de pacientes nefróticos $(\mathrm{n}=19)$ con un grupo control de pacientes no nefróticos $(n=41)$, encontrando que en el grupo estudio las pérdidas peritoneales eran significativamente mayores, lo que apoyaba la hipótesis de un efecto a nivel peritoneal del FP en pacientes nefróticos en DP ${ }^{19}$.

El objetivo de este estudio fue evaluar las pérdidas proteicas por peritoneo en pacientes con SN en diálisis peritoneal (DP), y compararlas con los pacientes en DP que no son portadores de Síndrome Nefrótico.

\section{Pacientes y Método}

Estudio descriptivo retrospectivo, observacional en los pacientes en diálisis peritoneal crónica controlados en la Unidad de Nefrología, Diálisis y Trasplante Renal del Hospital Luis Calvo Mackenna, período 2003-2007. Se consideró criterios de exclusión un episodio de peritonitis hasta 2 meses previo al ingreso, síndrome de malabsorción, diarrea crónica, enfermedades neurológicas, infecciones crónicas, uso de corticoides, enfermedades endocrinológicas, inhibidores de enzima convertidora, trastornos metabólicos, tratamientos con hormona de crecimiento y serología HIV/ HbsAg (+).

Se dividió a los pacientes en 2 grupos. El grupo en estudio (grupo 1) incluyó a los pacientes portadores de síndrome Nefrótico en DP. El grupo control (grupo 2) incluyó a los pacien- tes en DP con enfermedad renal secundaria a etiología no nefrótica, pareados por edad y sexo. La etiología del síndrome nefrótico fue diagnosticada por biopsia renal en todos los casos.

Se evaluaron los parámetros clínicos y de laboratorio seleccionados al primer mes y al $6^{\circ}$ ó $12^{\circ}$ mes de ingreso al programa en ambos grupos. Se registraron los siguientes antecedentes clínicos: edad (años), sexo, peso ( $\mathrm{kg}$ ), talla $(\mathrm{cm})$ y tiempo en DP (meses). Los datos bioquímicos incluyeron: creatinina sérica, nitrógeno ureico en plasma (BUN), calcio, fósforo, hormona paratiroidea intacta (IRMA-PTH), albúmina y proteínas plasmáticas. Se midieron las proteínas de 24 horas en el dializado y en orina al inicio y al final del período de seguimiento, éstas fueron medidas por test fotométrico usando rojo de pyrogallol en máquinas Hitachi 917 y 717 de nuestro hospital. El Test de equilibrio peritoneal (PET) ${ }^{20}$ fue evaluado al mes 2 y 6 de iniciada la DP. Se midió mensualmente el $\mathrm{KtV}$ peritoneal, y cada 3 meses el KtV residual. Se registraron: el volumen de dializado, la concentración de dextrosa, la diuresis diaria y la tasa de peritonitis. Si ocurría algún episodio de peritonitis, la evaluación de las pérdidas peritoneales se realizaba al menos 8 semanas después de completado el tratamiento antibiótico. Todos los pacientes recibieron alfa eritropoyetina y calcitriol de acuerdo a las guías DOQI ${ }^{21,22}$.

\section{Soporte nutricional}

Las indicaciones nutricionales correspondieron a las habituales en nuestros pacientes en DP, $100 \%$ de la ingesta energética diaria recomendada $(\mathrm{RDA})^{23}$ y las recomendaciones DOQI para la ingesta de proteínas ${ }^{24}$. Se realizó un monitoreo mensual de la ingesta proteica y energética. Los pacientes menores de 2 años fueron alimentados por medio de sonda nasogástrica o gastrostomía.

\section{Prescripción de diálisis}

La prescripción de diálisis está detallada en la tabla 2. Todos los pacientes recibieron diálisis intermitente automatizada (CCPD) Home Choice, Baxter ${ }^{\circledR}$, a volúmenes de $40-50 \mathrm{ml} / \mathrm{kg}$, para alcanzar un $\mathrm{Kt} / \mathrm{V}$ total $>2,1$. El volumen de dializado peritoneal de $24 \mathrm{hrs}$ fue recolecta- 
do en los mismos tiempos que los otros exámenes para la evaluación de la dosis de diálisis y las pérdidas proteicas. Se agregó timerosal a las muestras de orina y dializado para evitar la generación de urea secundaria a la actividad bacteriana.

\section{Análisis estadístico}

Los datos fueron reportados como promedios \pm desviación estándar. La diferencia de grupos fue calculada por $\mathrm{t}$ de student, un $\mathrm{p}<0,05$ fue considerado significativo.

\section{Resultados}

Se incluyeron 9 pacientes en el grupo en estudio, y 10 en el grupo control. Las características generales de ambos grupos se encuentran en la tabla 1. La etiología de los pacientes no nefróticos incluyó displasia renal, uropatías obstructivas y síndrome hemolítico urémico. Entre los pacientes con SN, 7 de ellos correspondieron a GEFS, y 2 a SN Congénito. No hubo diferencia significativa para ninguna de las características generales evaluadas entre los grupos control y nefróticos.

Las pérdidas proteicas peritoneales fueron
$3,41 \pm 2,01 \mathrm{gr} / \mathrm{m}^{2} /$ día para el grupo nefrótico $v s$ $1,76 \pm 1,45 \mathrm{gr} / \mathrm{m}^{2} /$ día para el grupo control al inicio del seguimiento $(\mathrm{p}<0,05), \mathrm{y} 4,27 \pm 3,5 v \mathrm{~s}$ $1,66 \pm 1,31 \mathrm{gr} / \mathrm{m}^{2} /$ día al final del estudio respectivamente $(\mathrm{p}<0.05)$, tabla 2 ; las pérdidas de proteínas en orina fueron $7,3 \pm 6,8 \mathrm{gr} / \mathrm{m}^{2} /$ día en el grupo nefrótico $v_{s} 0,3 \pm 0,2$ en el grupo control al ingreso $(\mathrm{p}<0,05)$, y $3,7 \pm 3,47$ vs 1,66 $\pm 1,31$ respectivamente al control del $12^{\circ}$ mes $(\mathrm{p}<0,05)$ (figuras 1 y 2 respectivamente). Los parámetros bioquímicos se detallan en la tabla 3. Sólo la PTH y la albúmina mostraron diferencias significativas en ambos grupos.

Para examinar el potencial efecto de la permeabilidad peritoneal y concentración de dextrosa de los baños en las pérdidas proteicas peritoneales, se calculó el PET analizando la relación dializado/plasma $(\mathrm{D} / \mathrm{P})$ de creatinina y $\mathrm{D}_{0} / \mathrm{D}_{4}$ de glucosa a las $4 \mathrm{hrs}$, y se comparó el tipo de solución dianeal ${ }^{\circledR}$ usado en ambos grupos en términos de la carga de dextrosa $(1,5$, $2,5$ y $4,25 \%)$. No se encontraron diferencias significativas tanto en la capacidad de depuración o ultrafiltración del peritoneo, como tampoco en la concentración de dextrosa utilizada en ambos grupos. Respecto a las peritonitis no hubo diferencias significativas en ambos grupos.

Tabla 1. Características generales de los pacientes en dialisis peritoneal portadores de síndrome nefrótico (grupo 1) y de otra etiología (grupo 2)

\begin{tabular}{|c|c|c|c|c|c|c|c|c|c|c|c|c|}
\hline \multirow[t]{2}{*}{ Paciente } & \multicolumn{2}{|c|}{$\begin{array}{c}\text { Edad al } \\
\text { ingreso (años) }\end{array}$} & \multicolumn{2}{|c|}{ Sexo } & \multicolumn{2}{|c|}{$\begin{array}{l}\text { Peso } \\
\text { (kg) }\end{array}$} & \multicolumn{2}{|c|}{$\begin{array}{l}\text { SC } \\
\left(m^{2}\right)\end{array}$} & \multicolumn{2}{|c|}{$\begin{array}{l}\text { Tiempo en DP } \\
\text { (meses) }\end{array}$} & \multicolumn{2}{|c|}{$\begin{array}{c}\text { Dianeal } \\
\% \text { dextrosa }\end{array}$} \\
\hline & G1 & G2 & G1 & G2 & G1 & G2 & G1 & G2 & G1 & G2 & G1 & G2 \\
\hline 1 & 12,4 & 0,3 & $\mathrm{~F}$ & $\mathrm{M}$ & 48,4 & 5,5 & 1,5 & 0,3 & 12 & 12 & $1,5 / 2,5$ & 1,5 \\
\hline 2 & 12,5 & 13,4 & $\mathrm{M}$ & $\mathrm{F}$ & 36,6 & 46,5 & 1,2 & 1,4 & 6 & 6 & $1,5 / 2,5$ & 1,5 \\
\hline 3 & 7,6 & 8,7 & $\mathrm{~F}$ & $\mathrm{M}$ & 22,7 & 25,2 & 0,9 & 0,9 & 12 & 6 & 1,5 & 1,5 \\
\hline 4 & 0,9 & 11,7 & $\mathrm{~F}$ & $\mathrm{~F}$ & 7,6 & 38,5 & 0,4 & 1,3 & 6 & 12 & 1,5 & 1,5 \\
\hline 5 & 0,5 & 1,5 & $\mathrm{~F}$ & $\mathrm{M}$ & 6,2 & 7,5 & 0,3 & 0,4 & 12 & 12 & $1,5 / 2,5$ & 1,5 \\
\hline 6 & 4,6 & 6,8 & $\mathrm{~F}$ & $\mathrm{~F}$ & 14,1 & 18,5 & 0,6 & 0,7 & 6 & 12 & 1,5 & 1,5 \\
\hline 7 & 9,8 & 2,3 & $M$ & $\mathrm{M}$ & 31 & 11 & 1,1 & 0,5 & 6 & 12 & $2,5 / 4,25$ & 1.5 \\
\hline 8 & 11,1 & 4 & $\mathrm{~F}$ & $\mathrm{~F}$ & 35 & 4,12 & 1,2 & 0,3 & 6 & 6 & $1,5 / 2,5$ & 1,5 \\
\hline 9 & 4,4 & 0,6 & $\mathrm{~F}$ & $\mathrm{M}$ & 15,1 & 8,35 & 0,6 & 0,4 & 12 & 12 & 1,5 & $1,5 / 2,5$ \\
\hline 10 & & 14,1 & & $\mathrm{M}$ & & 34,4 & & 1,2 & & 12 & & 2,5 \\
\hline Promedio & 7,1 & 6,3 & & & 24,1 & 20 & 0,9 & 0,8 & 8,7 & 10,2 & 2,1 & 1,7 \\
\hline Desv St & 4,7 & 5,4 & & & 14,5 & 15,3 & 0,4 & 0,4 & 3,2 & 2,9 & 0,8 & 0,4 \\
\hline
\end{tabular}

G1: Síndrome Nefrótico, G2: grupo control. 
Tabla 2. Tabla comparativa de los valores de dosis de diálisis (KtV), y proteínas en orina y dializado entre los grupos nefrótico y no nefrótico de niños en diálisis peritoneal crónica

\begin{tabular}{|c|c|c|c|c|c|c|}
\hline $\begin{array}{l}\text { Característica } \\
\text { (promedio } \pm \text { DS) }\end{array}$ & $\begin{array}{l}\text { Grupo } \\
\text { nefrótico } \\
\text { Inicio }\end{array}$ & $\begin{array}{l}\text { Grupo } \\
\text { control } \\
\text { Inicio }\end{array}$ & Valor $\mathbf{p}$ & $\begin{array}{l}\text { Grupo } \\
\text { nefrótico } \\
\text { Fin }\end{array}$ & $\begin{array}{c}\text { Grupo } \\
\text { control } \\
\text { Fin }\end{array}$ & Valor $\mathbf{p}$ \\
\hline KtV residual & $1,82 \pm 0,93$ & $2,39 \pm 1,24$ & Ns & $0,45 \pm 0,25$ & $1,38 \pm 1,05$ & $<0,05$ \\
\hline KtV peritoneal & $1,55 \pm 0,44$ & $1,69 \pm 0,76$ & Ns & $1,91 \pm 0,29$ & $1,73 \pm 0,91$ & Ns \\
\hline KtV total & $3,37 \pm 0,69$ & $4,08 \pm 1,50$ & Ns & $2,36 \pm 0,27$ & $3,11 \pm 0,98$ & Ns \\
\hline Proteínas en orina 24-h $\left(\mathrm{gr} / \mathrm{m}^{2}\right)$ & $7,3 \pm 6,8$ & $0,3 \pm 0,2$ & $<0,05$ & $3,7 \pm 2,3$ & $0,65 \pm 0,5$ & $<0,05$ \\
\hline Proteínas dializado 24-h $\mathrm{gr} / \mathrm{m}^{2}$ ) & $3,41 \pm 2,01$ & $1,76 \pm 1,45$ & $<0,05$ & $4,27 \pm 3,47$ & $1,66 \pm 1,31$ & $<0,05$ \\
\hline
\end{tabular}

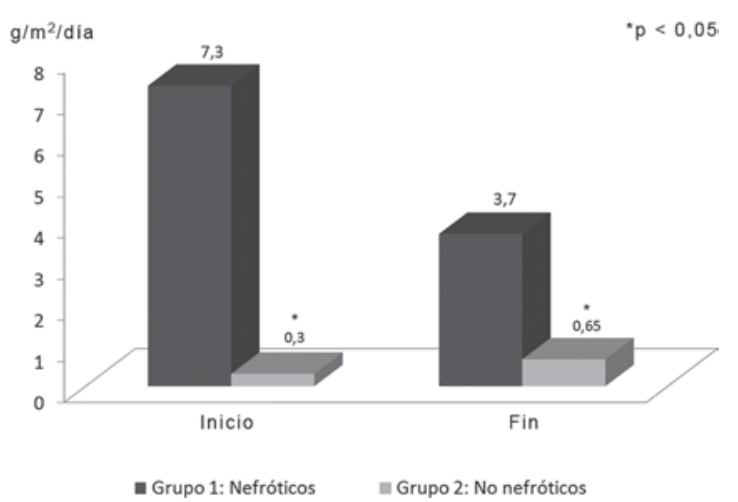

Figura 1. Pérdidas proteicas urinarias.

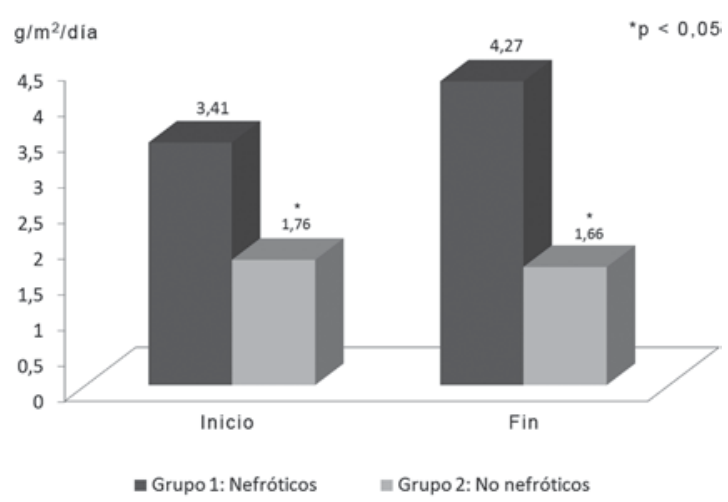

Figura 2. Pérdidas proteicas peritoneales.

Tabla 3. Tabla comparativa de los valores de exámenes bioquímicos plasmáticos entre los grupos nefrótico y no nefrótico de niños en diálisis peritoneal crónica

\begin{tabular}{|c|c|c|c|c|c|c|}
\hline $\begin{array}{l}\text { Característica } \\
\text { (promedio } \pm \text { DS) }\end{array}$ & $\begin{array}{l}\text { Grupo } \\
\text { nefrótico } \\
\text { Inicio }\end{array}$ & $\begin{array}{l}\text { Grupo } \\
\text { Inicio }\end{array}$ & Valor $\mathbf{p}$ & $\begin{array}{c}\text { Grupo } \\
\text { nefrótico } \\
\text { Fin }\end{array}$ & $\begin{array}{c}\text { Grupo } \\
\text { control } \\
\text { Fin }\end{array}$ & Valor $p$ \\
\hline Creatinina (mg/dl) & $3,19 \pm 1,77$ & $4,64 \pm 3,21$ & Ns & $6,76 \pm 2,09$ & $4,88 \pm 3,01$ & ns \\
\hline Nitrógeno uréico (mg/dl) & $42,1 \pm 18,4$ & $42,1 \pm 21,9$ & Ns & $54,1 \pm 13,6$ & $47 \pm 15,7$ & ns \\
\hline Bicarbonato (mEq/L) & $22,5 \pm 3,1$ & $24,2 \pm 5,0$ & Ns & $24,4 \pm 4,7$ & $23,1 \pm 3,1$ & ns \\
\hline Hemoglobina ( $\mathrm{gr} / \mathrm{dl})$ & $9,2 \pm 1,2$ & $10,6 \pm 2,2$ & Ns & $10,4 \pm 1,5$ & $11,7 \pm 2,2$ & ns \\
\hline Calcio (mg/dl) & $8,9 \pm 1,3$ & $8,9 \pm 2,8$ & Ns & $9,2 \pm 2,3$ & $9,5 \pm 0,9$ & ns \\
\hline Fósforo (mg/dl) & $5,2 \pm 1,4$ & $5,5 \pm 1,6$ & Ns & $5,3 \pm 1,2$ & $5,6 \pm 1,4$ & ns \\
\hline Fosfatasas alcalinas (U/L) & $294,3 \pm 146,2$ & $428 \pm 263,4$ & Ns & $323 \pm 182,7$ & $474,8 \pm 219,7$ & ns \\
\hline Paratohormona (pg/ml) & $227 \pm 198$ & $360 \pm 324$ & Ns & $164 \pm 144$ & $564 \pm 454$ & $<0,05$ \\
\hline Albúmina silica (gr/dl) & $2,27 \pm 0,63$ & $3,62 \pm 1,45$ & $<0,05$ & $2,8 \pm 0,5$ & $3,9 \pm 0,86$ & $<0,05$ \\
\hline
\end{tabular}

\section{Discusión}

El síndrome nefrótico se caracteriza por el aumento de la permeabilidad de la membrana basal glomerular a las proteínas plasmáticas generando proteinuria que lleva al paciente a la hipoalbuminemia.
Desde 1974 se describe la existencia de un factor de permeabilidad presente en algunas formas de SN y que sería responsable de las recaídas de la enfermedad sobre el injerto renal en etapas post-trasplante ${ }^{18,25-32}$. Kopanati fue el primer autor en estudiar el posible efecto del FP en la membrana peritoneal midiendo las 
pérdidas proteicas en el dializado de pacientes con $\mathrm{SN}$, encontrando una pérdida casi el doble de lo medido en pacientes en DP por otras causas de IRC $^{19}$. Al igual que el estudio de Kopanati, las diferencias en las pérdidas proteícas peritoneales encontradas en nuestro protocolo fueron significativamente mayores en el grupo de pacientes con $\mathrm{SN}$, tanto al ingreso como al final de la evaluación. Como era esperable, las pérdidas urinarias mostraron la misma relación que en peritoneo. Un factor que puede producir distorsión en la cantidad de proteínas presentes en el dializado es la dosis de diálisis aplicada a cada paciente; en este sentido se evaluó el $\mathrm{KtV}$ peritoneal y total durante todo el seguimiento, no encontrándose diferencias significativas entre los 2 grupos. Lo mismo debe aplicarse a la concentración del dianeal ${ }^{\circledR}$ utilizado y a las tasas de peritonitis, variables que potencialmente pueden influir en la pérdida proteica peritoneal, que en este estudio no mostraron diferencias significativas entre los grupos (tabla 1). Si bien en el estudio de Kopanati destaca que el grupo de pacientes nefróticos tenían edad mayor que el grupo control, en nuestro estudio no hubo diferencias entre los grupos y para eliminar el efecto que tendría comparar las pérdidas proteicas peritoneales en pacientes de distintas superficies corporales se estandarizaron las pérdidas proteicas por metro cuadrado de superficie corporal, y al igual que el autor antes mencionado encontramos que los pacientes nefróticos tienen pérdidas proteicas peritoneales cercano al doble de los pacientes no nefróticos en DP.

La alteración de la permeabilidad peritoneal en pacientes con síndrome nefrótico en diálisis ha sido igualmente atribuida a factores mecánicos. La filtración de moléculas a través del peritoneo es explicada por el modelo de los tres poros, definido por la permeabilidad asociada al tamaño de estos poros de filtración ${ }^{33}$. Basándose en este principio, De Boer y cols, estudiaron la permeaselectividad de la membrana peritoneal en niños con SN en DP midiendo proteínas de distinto peso molecular en el dializado: $\beta 2$ microglobulina (PM11800D), albúmina (PM 69000D), IgG (PM 160000D) y $\alpha 2$ macroglobulina (PM820000D), sin encontrar diferencias significativas a nivel peritoneal en ninguna de las proteínas estudiadas entre los 2 grupos, alejando la posibilidad de posibles alteraciones mecánicas de la membrana peritoneal de estos pacientes ${ }^{9}$. Esto apoya más el hecho de que las mayores pérdidas peritoneales encontradas en pacientes nefróticos en DP pudiesen ser atribuidas a un factor soluble de permeabilidad capilar, más que a alteraciones mecánicas de la barrera de filtración del peritoneo.

La importancia del FP continúa en investigación, uno de los FP identificados a los que se le ha prestado mayor atención es al identificado por Savin y Sharma, descrito inicialmente en glomérulos cultivados de rata, y asociado posteriormente con un potencial riesgo de recurrencia del SN post-trasplante en pacientes con GEFS. Se ha logrado identificar y medir la actividad del FP en glomérulos aislados de pacientes con SN y se ha descrito como una proteína de bajo peso molecular que puede ser filtrada por la barrera de filtración aniónica ${ }^{25}$. Se ha postulado la presencia de FP incluso en pacientes trasplantados renales portadores de SN Finés, como asimismo en pacientes trasplantados portadores del síndrome nefrótico genético por mutación del gen de la podocina (NPHS2), evidenciando que las recaídas del síndrome nefrótico postrasplante en estos casos pueden asociarse a la acción del FP en el órgano trasplantado ${ }^{30-31}$. Se ha descrito además que este FP puede ser removido por inmunoabsorción de proteína $\mathrm{A}$, que circula en asociación con $\operatorname{IgG}^{34}$, que cruza la placenta y puede inducir proteinuria neonatal transitoria ${ }^{35}$. En la búsqueda de sustancias que puedan contrarrestar el efecto de este FP se observó in vitro que la orina de pacientes nefróticos corregía la permeabilidad del glomérulo nefrótico, lo que no ocurría con orina de pacientes normales, por lo que los pacientes nefróticos deben excretar una sustancia inhibidora del FP por orina. Candiano y cols ${ }^{36}$, sugieren que algunos componentes de las proteínas de alta densidad plasmáticas de suero de sujetos sanos como son las apolipoproteínas E2, E4, J, L y A-IV inhiben el FP in vitro de glomérulos incubados con suero de pacientes con GEFS in vitro, así como preparados comerciales de Apo E y J, postulando que en pacientes con GEFS existiría un déficit de inhibidores del FP. En una reciente 
comunicación de Savin y cols, la terapia con galactosa se ha utilizado con éxito en el tratamiento de un paciente con recaída de GEFS post-trasplante, postulando que el FP puede interactuar con azúcares del glicocálix, y al medir la actividad del FP posterior al uso de galactosa se evidenció la pérdida de actividad de este factor, desde 0,88 previo a la infusión de galactosa a niveles indetectables a las $48 \mathrm{hrs}$ de infusión, manteniéndose bajos niveles de FP al menos por 4 semanas después de suspendida la galactosa. Se ha propuesto así a la galactosa como una nueva terapia de tratamiento para pacientes con GEFS que podría actuar tanto en la prevención de progresión de la enfermedad como en las recaídas post trasplante ${ }^{37}$.

En conclusión, el SN en pediatría es una enfermedad de etiología multifactorial, entre cuyas causas la existencia de un FP de probable origen en el sistema inmune, que representa una vía etiopatogénica de alta importancia en el manejo de los casos primarios y postrasplante renal. En este estudio se ratifican los hallazgos descritos previamente por Kopanati que apoya la hipótesis de que en niños portadores de SN en diálisis peritoneal, la persistente hipoalbuminemia y malnutrición pudiesen estar asociadas a una mantenida y exacerbada pérdida de proteínas vía peritoneal. Parece así ser de alta importancia la determinación de este FP, tanto desde un punto de vista diagnóstico como terapéutico durante la etapa de diálisis como en la etapa postrasplante renal. Se requieren futuros estudios que involucren mayor número de pacientes y técnicas que permitan medir directamente el FP en estos niños, para dar pasos decisivos en una enfermedad tan antigua como desafiante, como es el Síndrome Nefrótico.

\section{Referencias}

1.- Eddy AA, Symons JM: Nephrotic syndrome in childhood. Lancet 2003; 362: 629-39.

2.- Hodson E: The management of idiopathic nephrotic syndrome in children. Paediatr Drugs 2003; 5: 335 49.

3.- Vogel A, Azócar M, Nazal V: Actualizaciones en el tratamiento del Síndrome Nefrótico Idiopático. Recomendaciones de la Rama de Nefrología de la Sociedad
Chilena de Pediatría. Rev Chil Pediatr 2006; 77 (3); 295-303.

4.- ISKDC: The primary nephrotic syndrome in children: prediction of Histopathology from clinical and laboratory characteristics at time of diagnosis. A report of the International Study of Kidney disease in Children. Kidney Int 1978; 13: 159-65.

5.- D'Agati $V$ : The many masks of focal segmental glomerulosclerosis. Kidney Int 1994; 46: 1223-41.

6.- Haas M, Spargo BH, Coventry S: Increasing incidence of focal-segmental glomerulosclerosis among adult nephropathies: a 20-year renal biopsy study. Am J Kidney Dis 1995; 26: 740-50.

7.- Haas M, Meehan SM, Karrison TG, Spargo BH: Changing etiologies of unexplained adult nephrotic syndrome: a comparison of renal biopsy findings from 1976-1979 and 1995-1997. Am J Kidney Dis 1997; 30: 621-63.

8.- Srivastava T, Simon SD, Alon US: High incidence of focal segmental glomeruloesclerosis in nephrotic syndrome of childhood. Pediatr Nephrol 1999; 13: 13-8.

9.- De Boer AW, Schröder CH, Reddingius RE, Willems $H L$, Monnens LA: Peritoneal protein loss in children with nephrotic syndrome during peritoneal dialysis. Nephrol Dial Transplant 1998; 13: 2348-50.

10.- NAPTRCS: web.emmes.com/study/ped/annlrept/ annlrept2006.pdf. 2006

11.- Azócar MA, Cano FJ, Marín V, Delucchi MA, Rodríguez $E E$ : Body composition in children on peritoneal dialysis. Adv Perit Dial 2004; 20: 231-6.

12.- Warady BA, Alexander SR, Watkins S, Kohaut E, Harmon WE: Optimal care of the pediatric end-stage renal disease patient on dialysis. Am J Kidney Dis 1999; 33 (3): 567-83.

13.- Cano FJ, Marín VS, Azócar MA, Delucchi MA, Rodríguez EE, Díaz ED, et al: Adequacy and nutrition in pediatric peritoneal dialysis. Adv Perit Dial 2003; 19: 273-8.

14.- Rees L, Shaw V: Nutrition in children with CRF and on dialysis. Pediatr Nephrol 2007; 22: 1689-702.

15.- Stiles KP, Abbott KC, Welch PG, Yuan CM: Effects of angiotensin converting enzyme inhibitor and steroid therapy on proteinuria in FSGS: a retrospective study in a single clinic. Clin Nephrol 2001; 56: 89-95.

16.- Tabel Y, Berdeli A, Mir S, Serdaroglu E, Yilmaz E: Effects of genetic polymorphisms of the reninangiotensin system in children with nephrotic syndrome. J Renin Angiotensin Aldosterone Syst 2005; 6 (3): 138-44. 
17.- Zúñiga $Z V$, Ellis D, Moritz ML, Docimo SG: Bilateral laparoscopic transperitoneal nephrectomy with early peritoneal dialysis in an infant with the nephrotic syndrome. J Urol 2003; 170 (5): 1962.

18.- Shalhoub A RJ: Pathogenesis of lipoid nephrosis: A disorder of T-cell function. Lancet 1974; 2: 556-60.

19.- Kopanati S, Baum M, Quan A: Peritoneal protein losses in children with steroid-resistant nephrotic syndrome on continuous-cycler peritoneal dialysis. Pediatr Nephrol 2006; 21: 1013-9.

20.- Twardowski ZJ: Peritoneal equilibration test. Perit Dial Bull 1987; 3: 138-47.

21.- KDOQI: Clinical Practice Guidelines and Clinical Practice Recommendations for Anemia in Chronic Kidney Disease. Am J Kidney Dis 2006; 47: S1-S146, (suppl 3).

22.- K/DOQI: Clinical Practice Guidelines for Bone Metabolism and Disease in Children with Chronic Kidney Disease. Am J Kidney Dis 2005; 46: S1-S122, (suppl 1).

23.- Food and Nutrition Board, Commission on Life Sciences, National Research Council: Recommended Dietary Allowances (ed 10). Washington, DC, National Academy Press, 1989.

24.- http://www.kidney.org/professionals/Kdoqi/ guidelines_updates/nut_p06.htm

25.- Savin VJ, Sharma R, Sharma $M$, et al: Circulating factor associated with increased glomerular permeability to albumin in recurrent focal segmental glomerulosclerosis. N Engl J Med 1996; 334: 878-83.

26.- Feld SM, Figueroa P, Savin V, et al: Plasmapheresis in the treatment of steroid-resistant focal and segmental glomerulosclerosis in native kidneys. Am J Kidney Dis 1988; 32: 230-7.

27.- Artero ML, Sharma R, Savin VJ, Vincenti F: Plasmapheresis reduces proteinuria and serum capacity to injure glomeruli in patients with recurrent focal glomerulosclerosis. Am J Kidney Dis 1994; 23: 574-81.
28.- Sharma M, Sharma R, Reddy SR, McCarthy ET, Savin $V J$ : Proteinuria after injection of human focal segmental glomerulosclerosis factor. Transplantation 2002; 73: 366-72.

29.- Meyrier A: Nephrotic focal segmental glomeruloesclerosis in: an update. Nephrol Dial Transplant 2004; 19: 2437-44.

30.- Srivastava T, Garola R, Kestila M, et al: Recurrence of proteinuria following renal transplantation in congenital nephrotic syndrome of the Finnish type. Pediatr Nephrol 2006; 21: 711-8.

31.- Carraro M, Caridi G, Bruschi $M$, et al: Serum glomerular permeability activity in patients with podocin mutations (NPHS2) and steroid-resistant nephritic syndrome. J Am Soc Nephrol 2002; 13: 1946-52.

32.- Flynn JT, Schulman SL, Dechadarevian JP, et al: Treatment of steroidresistant post-transplant nephrotic syndrome with cyclophosphamide in a child with congenital nephrotic syndrome. Pediatr Nephrol 1992; 6: 553-5.

33.- Flessner MF: Peritoneal transport physiology: insights from basic research. J Am Soc Nephrol 1991; 2: 12235 .

34.- Esnault VL, Besnier D, Testa A, et al: Effect of protein A immunoadsorption in nephrotic syndrome of various etiologies. J Am Soc Nephrol 1999; 10: 2014-7.

35.- Kemper MJ, Wolf G, Muller-Wiefel DE: Transmission of glomerular permeability factor from a mother to her child. N Engl J Med 2001; 344: 386-7.

36.- Candiano $G$, Musante L, Carraro $M$, et al: Apolipoproteins prevent glomerular albumin permeability induced in vitro by serum from patients with focal segmental glomerulosclerosis. J Am Soc Nephrol 2001; 12: $143-50$.

37.- Savin VJ, Mccarthy ET, Sharma R, Charba D, Sharma $M$ : Galactose binds to focal segmental glomeruloesclerosis permeability factor and inhibits its activity. Transl Res 2008; 151 (6): 288-92. 